\title{
Management of Nonurological Pelvic Tumors Infiltrating the Lower Urinary Tract
}

\author{
Claudius Falch • Bastian Amend · Sven Müller • Andreas Kirschniak • \\ Ruth Ladurner • Beate Wietek · Karl-Dietrich Sievert • Christl Reisenauer • \\ Alfred Königsrainer
}

Published online: 11 October 2014

(C) Springer Science + Business Media New York 2014

\begin{abstract}
Locally advanced tumors, originating from organs and structures of the pelvis and the pelvic floor may compromise the lower urinary tract. The aim of this review is to display the diagnosis and treatment of the most common malignancies infiltrating the lower urinary tract. A review was performed of the literature in PubMed over the last two decades. The most common nonurological pelvic tumors infiltrating the lower urinary tract are colorectal cancer, gynecological tumors, and sarcomas. For diagnosis, multiple non-invasive and invasive options are available and their use is partially tumor-specific. If infiltration to adjacent structures is given, en bloc resection should be performed to
\end{abstract}

This article is part of the Topical Collection on Prostate Surgery and Related Procedures.

\footnotetext{
C. Falch · S. Müller · A. Kirschniak · R. Ladurner .

A. Königsrainer $(\varangle)$

Department of General, Visceral, and Transplant Surgery,

Tübingen University Hospital, Hoppe-Seyler-Strasse 3,

72076 Tübingen, Germany

e-mail: alfred.koenigsrainer@med.uni-tuebingen.de

C. Falch

e-mail: claudius.falch@med.uni-tuebingen.de

S. Müller

e-mail: sven.mueller@med.uni-tuebingen.de

A. Kirschniak

e-mail: andreas.kirschniak@med.uni-tuebingen.de

R. Ladurner

e-mail: ruth.ladurner@med.uni-tuebingen.de

C. Falch - B. Amend - S. Müller - A. Kirschniak - R. Ladurner ·

B. Wietek - K.-D. Sievert · C. Reisenauer - A. Königsrainer University of Tübingen: Comprehensive Cancer Center and Continence and Pelvic Floor Center, Tübingen, Germany

e-mail: bastian.amend@med.uni-tuebingen.de
}

achieve clear margins. However, despite oncological considerations a bladder-sparing resection is often possible. Neoadjuvant or adjuvant therapy approaches are also tumorspecific. The overall 3-year survival rate is about $40 \%$. Nonurological tumors that infiltrate the lower urinary tract should be given multidisciplinary treatment. The patient's personal decision regarding acceptance of the surgical extent with possibly persistent disability should be considered.

Keywords Nonurological pelvic tumors - Multivisceral resection $\cdot$ Bladder-sparing surgery $\cdot$ Colorectal cancer . Pelvic floor
B. Wietek

e-mail: beate.wietek@med.uni-tuebingen.de

K.-D. Sievert

e-mail: karl.sievert@med.uni-tuebingen.de

C. Reisenauer

e-mail: christl.reisenauer@med.uni-tuebingen.de

B. Amend · K.-D. Sievert

Department of Urology, Tübingen University Hospital,

Hoppe-Seyler-Strasse 3, 72076 Tübingen, Germany

B. Wietek

Department of Diagnostic and Interventional Radiology,

Tübingen University Hospital, Hoppe-Seyler-Strasse 3,

72076 Tübingen, Germany

C. Reisenauer

Department of Gynecology, Tübingen University Hospital,

Calwerstrasse 7, 72076 Tübingen, Germany 


\section{Introduction}

Locally advanced tumors originating from organs and structures of the pelvis and the pelvic floor may compromise the lower urinary tract by infiltration, compression, or displacement and thereby become symptomatic. The most common nonurological pelvic tumors infiltrating the lower urinary tract are colorectal cancer, gynecological tumors, and sarcomas [1••]. Often, tumor infiltration cannot be distinguished from inflammatory adhesions preoperatively or intraoperatively [2]. Therefore, a multidisciplinary approach is necessary, and accurate tumor staging and treatment planning are significant. The aim of this review is to describe the most common tumor entities that can affect the lower urinary tract as well as pre- and intraoperative surgical treatment options including reconstruction of the lower urinary tract.

\section{Methods}

A review was conducted of the literature (PubMed, Cochrane Library) published over the last two decades with regard to diagnosis and treatment of tumors in the small pelvis with infiltration of the lower urinary tract. The Cochrane Library permits no systematic review of these topics.

\section{Diagnostics and Imaging}

\section{General Aspects of Diagnostics}

Patients suffering from tumor infiltration of the urinary tract may present with hematuria, urinary tract infection, urinary incontinence, obstruction of the lower or upper urinary tract, and with renal insufficiency in cases of chronic hydronephrosis [3]. The possibility of a urogenital tumor, e.g., transitional cell carcinoma of the urinary bladder, should be kept in mind if histological examination of the tumor has not yet been performed [3-5].

\section{Laboratory Testing}

Urine dipstick analysis is most likely the basic "key examination" to detect microhematuria. Painless (micro-) hematuria is one of the most frequent complaints of patients with bladder cancer [4] and urinary tract infiltration by nonurological cancer. Chuttiangtum et al. reported a study involving 130 cervical cancer patients; $41.5 \%$ demonstrated a positive dipstick analysis and four patients had pathologically confirmed infiltration of the bladder. Sensitivity was calculated to be $100 \%$ with a specificity of
$60.3 \%$. The authors concluded that hematuria might be a good tool for deciding whether further examination (e.g., cystoscopy) is necessary [6].

In addition to hematuria, dipstick analysis also focuses on urinary tract infection. Infection can be only a concomitant problem, but especially patients with recurrent, treatment-resistant urinary tract infections, or typical enterobacteria might already suffer from a fistula to either the intestine or the female inner genitalia [5].

Urine cytology is well established in the detection and oncological follow-up of carcinoma of the urinary bladder and the upper urinary tract [7]. Snyman et al. described the urine cytology for nonurological tumors as having a sensitivity of $94 \%$ and a specificity of $82 \%$ [8]. In accordance with Chuttiangtum et al. [6] they indicated invasive investigation of the urinary tract by cystoscopy in patients with positive cytology.

\section{Ultrasound}

Ultrasound is a helpful tool for detecting possible impairment of the upper urinary tract and macroscopic tumor involvement of the urinary bladder.

Hydronephrosis in patients with pelvic tumors is usually a sign of advanced disease with ureteric infiltration [5, 9••]. In cases of hydronephrosis the thickness of kidney parenchyma may be an important criterion for kidney function [10]. If chronic hydronephrosis is suspected or renal parenchyma thickness is considerably reduced, MAG-3 renal scintigraphy should be performed to determine residual renal function and ultimately assess organ preservation. With regard to renal impairment and the avoidance of iatrogenic intraoperative ureteric trauma, retrograde urography with double-J stenting or, if not possible, placement of a nephrostomy tube should be considered [5].

Both transabdominal and transrectal ultrasound examinations are of little value in diagnosis and staging, even though transvaginal ultrasound has been shown to be of some benefit in patients with advanced cervical cancer $[11,12]$.

\section{Cystography and Cystoscopy}

Cystoscopy remains the gold standard for evaluation of the lower urinary tract if tumor infiltration is suspected [4]. Flexible cystoscopes, videoendoscopy, and digital recording permit quick examination [13]. Simple forceps biopsies are generally feasible under local anesthesia. If resection biopsies or deep biopsies are necessary, general or spinal anesthesia is required. Figure 1 shows a cystoscopic view of intramural tumor masses with transurethral resection biopsies.

When ureteral obstruction and/or a vesicointestinal or vesicogenital fistula are found, ureteric stenting is possible. 
Fig. 1 a Cystoscopic view of the bladder trigone with the left ureteral orifice stented with a double-J catheter. Medial to the orifice the bladder mucosa demonstrates alterations after irradation therapy decades before and elevation due to impression/invasion of a supravaginal tumor. b Same view after transurethral resection (biopsy) with intramural solid tumor mass
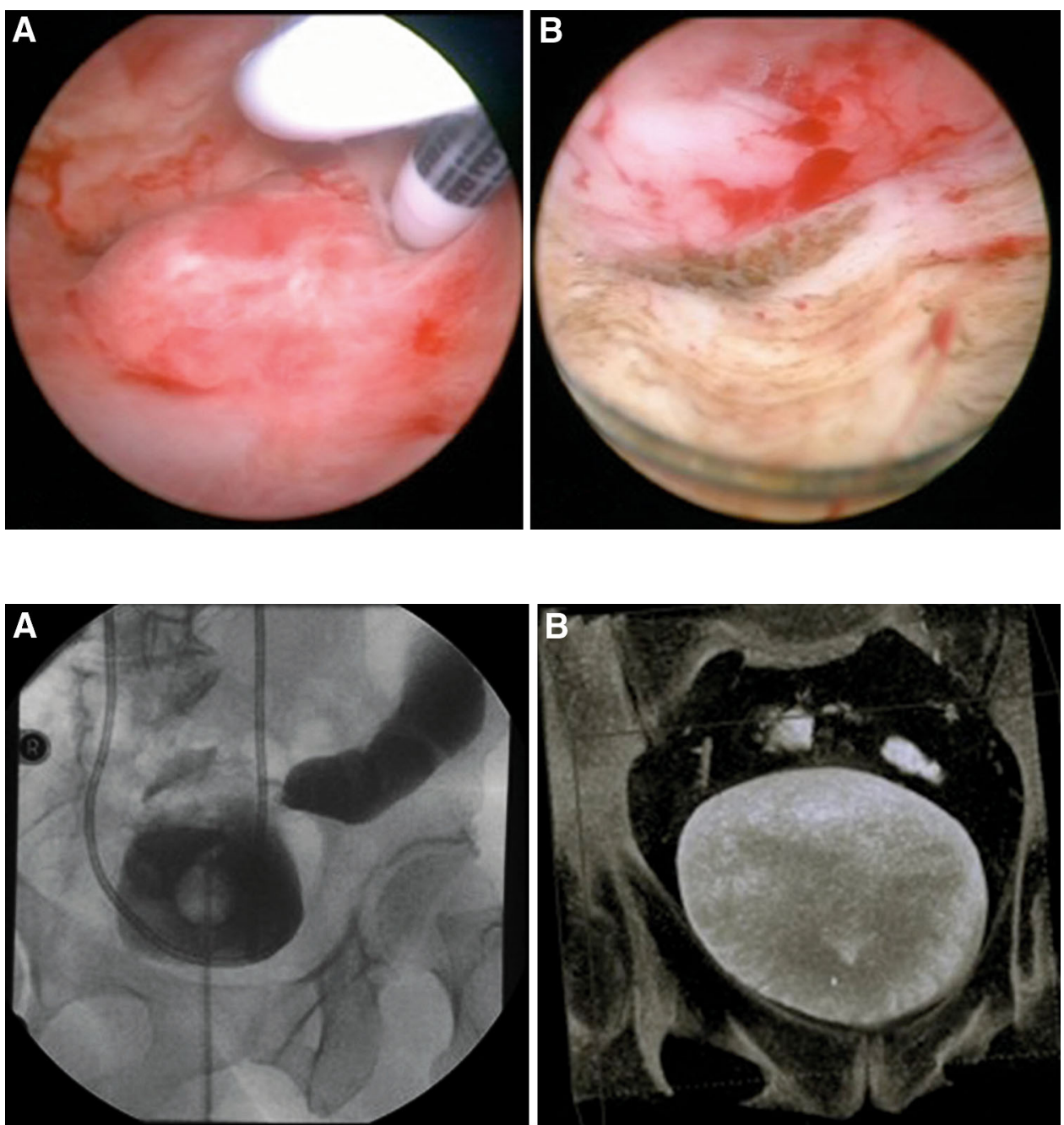

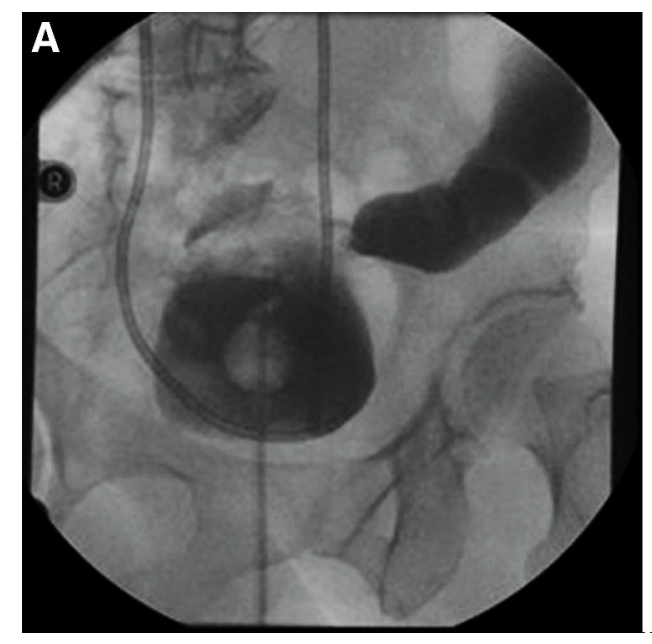

Fig. 2 a Conventional X-ray cystography. Bladder is filled with contrast medium through a transurethral catheter. Double-J stents are visible in projection of both ureters. Vesicointestinal fistula causes contrast accumulation in the sigmoid colon. b 3D reconstruction of a cystography (different patient) performed with conventional X-ray (MultiDiagnost ELEVA, Philips GmbH, Hamburg, Germany). Contrast medium passes through a small fistula into the colon
Figure 2 illustrates conventional cystography for the identification of a broad fistula and 3D conventional X-ray reconstruction for topographic illustration of the fistula. The greatest sensitivity for fistula detection seems to be provided by the poppy seed test $(94.8 \%)$, whereas imaging and cystoscopy have demonstrated considerably less sensitive results (CT scan $58.7 \%$, cystography $19.4 \%$, cystoscopy $15.1 \%$, and colon enema $38.6 \%$ ) [14].

\section{Tomographic Imaging}

Owing to its superior soft-tissue contrast resolution and multiplanar capability, magnetic resonance imaging (MRI) is now a widely used imaging modality for the pelvis, especially in the initial primary tumor staging of advanced cervical as well as endometrial and rectal cancer.

Computed tomography (CT) is currently the best imaging technique for staging ovarian cancer [15]. CT provides rapid image acquisition with an overall accuracy of $70-90 \%$ for the detection of tumors at all stages. The most serious limitation of CT is its inability to show nodules with a maximum diameter of less than $5 \mathrm{~mm}$. The detection rate for such small lesions is poor with sensitivities of only 7-50 \% [16]. CT and MRI techniques have comparable overall sensitivities for the detection of peritoneal deposits (92 vs. $95 \%$ ) [17]. Contrast-enhanced MRI and additional functional imaging techniques may allow diagnostic sensitivities greater than those achieved with CT. Especially diffusion-weighted imaging (DWI) and contrast-enhanced (CE) MRI may improve the accuracy of tumor detection, in particular for the assessment of pelvic organs and musculature [18].

Recently, Positron emission tomography-computed tomography (PET-CT) was introduced as a diagnostic tool for patients with ovarian cancer, advanced gynecological cancer as well as with recurrent disease. In a recent metaanalysis PET-CT was shown to play an important role in the evaluation of recurrent disease with a highest pooled sensitivity (92\%) for ovarian cancer as compared with PET or CT or MRI alone [19]. In patients with advanced 
Fig. 3 a CT image, b fused PET-CT image in axial plane, c PET image, $\mathbf{d}$ sagittal PET image. Female patient (24y) with ovarian cancer, adnexectomy and hysterectomy. After 1 year recurrent disease with infiltration of sigmoid, rectum and dorsal vesical wall. Additional lymph node involvement at the left iliac artery as well as a solid metastasis in the ventral pelvic wall are present
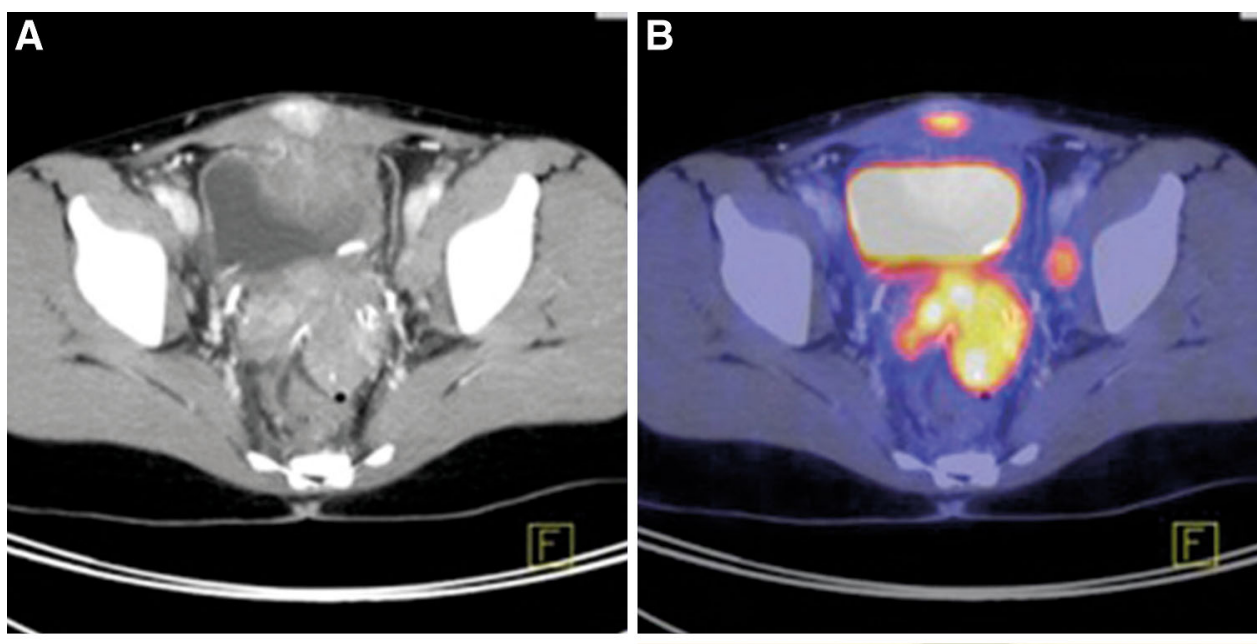

C

D

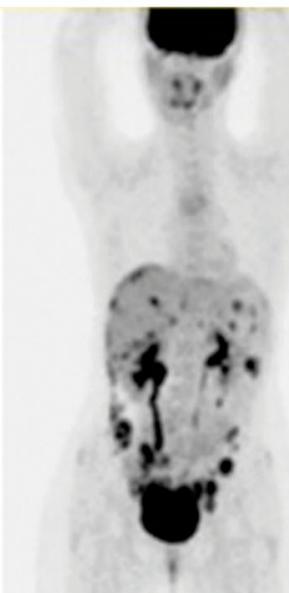

disease at presentation, PET-CT has been found to alter management in a significant number of patients (up to $65 \%)$ [20].

MR-PET combines the advantages of both techniques in the detection of tumor invasion of pelvic organs and/or the pelvic floor.

Figures 3 and 4 illustrate examples of clinical application of CT, PET, and PET-CT. Figure 5 shows the same for MRI, PET, and PET-MRI.

\section{Etiology, Behavior and Therapy}

Tumors Originating from the Lower Gastrointestinal Tract

Adenocarcinomas of the colon and rectum (CRC) are the most common malignancies of the lower gastrointestinal tract (LGIT). Surrounding structures are infiltrated in approximately 10-20\% of cases [21]. Involvement of the lower urinary tract arises mainly from primary or recurrent tumors of the colon sigmoideum or the rectum $[1 \bullet \bullet, 22]$. Tumor recurrence of CRC is reported to be as high as $14-43 \%$ if the lower urinary tract seems to be infiltrated $[1 \bullet, 23 \bullet, 24]$. If the lower urinary tract is involved, most commonly the left ureter, bladder dome, supratrigonal, and bladder neck are affected [1••]. In up to $80 \%$ of the patients, acute urinary retention, hematuria, or fecaluria are the first symptoms. [1・•]. The type of preoperative diagnosis follows the same guidelines as for staging of rectal cancer. [25•].

In primary rectal cancer with infiltration of adjacent structures (T4 situation), downsizing and a higher rate of clear margins can be achieved significantly more often by neoadjuvant chemoradiation [26] or short-term irradiation [25•]. If no neoadjuvant therapy was performed in the primary resection of rectal cancer, this should be done in cases of local recurrence [25•]. If R0 resection is possible in previously treated patients, primary resection should be performed [25•]. Different constellations require different approaches $[25 \bullet$.

Discrimination between tumor-related inflammatory adhesions and infiltration is usually not possible [2]. In 
Fig. 4 a CT image, b fused PET-CT image in axial plane, c PET image, $\mathbf{d}$ sagittal PET image with marked hydronephrosis. Female patient (63y) with endometrial cancer, post-hysterectomy and radiochemotherapy after an ileus operation with colostomy and Hartmann stump. After 3 years advanced recurrence with infiltration of the Hartmann stump and infiltration of the vesical with marked hydronephrosis
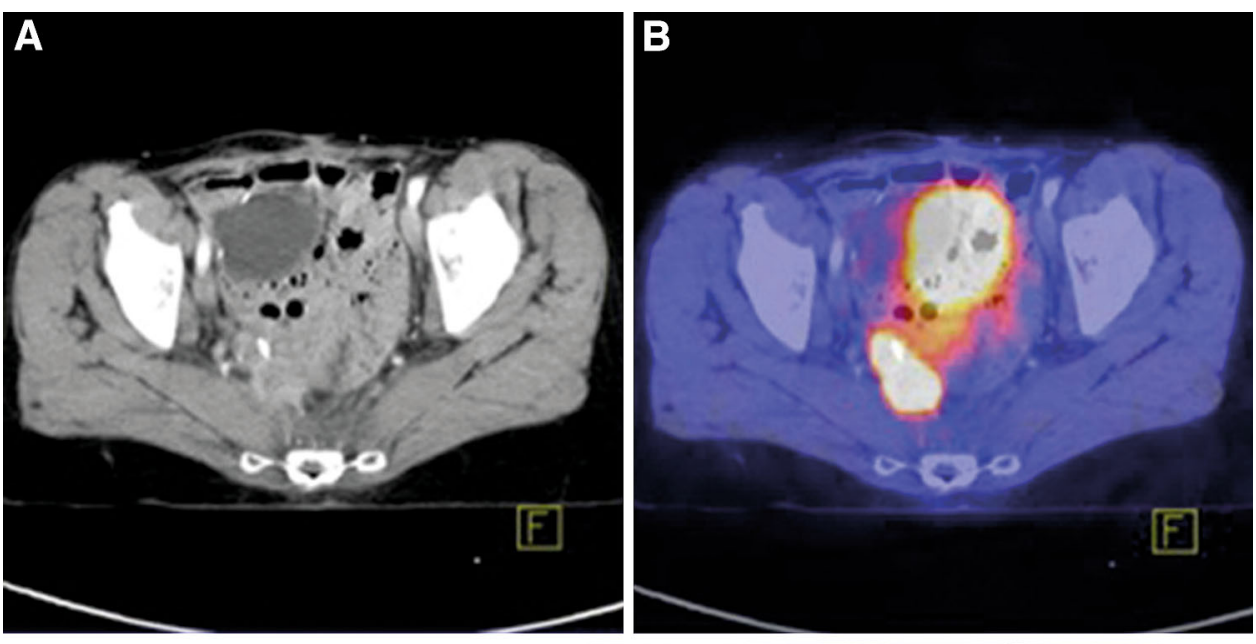

C
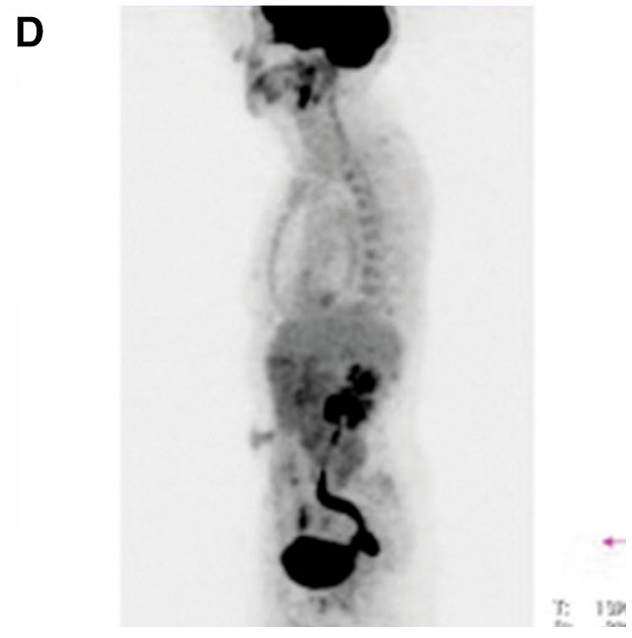

published collectives, the ratio of tumor-related infiltration to inflammatory adhesions ranges from 54.1-66.7 \% to $33.3-45.9 \%[1 \bullet, 2]$. The risk of tumor cell dissemination means biopsies $[25 \cdot, 27]$ and dissection or separation of the

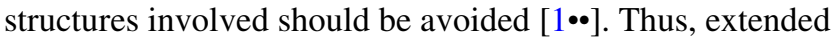
en bloc resections are recommended [25•].

Tumor infiltration due to CRC is mostly unifocal with the

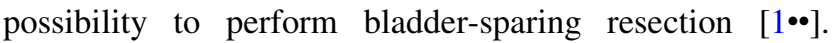
Without influencing the oncological outcome or overall survival, this limited resection results in improved postoperative quality of life $[1 \bullet \bullet]$. The most important predictor for locoregional tumor control and survival for primary locally advanced CRC as well as for locally recurrent CRC is a complete resection (R0) [21, 28]. To achieve this, multivisceral resection and even complete pelvic exenteration including sacrectomy might be necessary [28]. This highlights the need for a multidisciplinary approach $[23 \bullet, 28]$.

Postoperative complications occur less frequently after bladder-sparing resection than after extended resection or cystectomy (10.5-29\% vs. 37-58.3\%) [23•, 24]. Surgery for locally recurrent CRC is also associated with a higher rate of comorbidity than is surgery for primary CRC [21]. Urologic incontinence symptoms (frequency, urgency, and urge incontinence) are described in up to $50 \%$ of patients 1 year after surgery [1••]. These can be explained by a smaller bladder volume after resection and injury to the pelvic nerve plexus $[1 \bullet \bullet]$.

The three-year survival rate for primary locally advanced CRC with infiltration into the lower urinary tract depends on the primary tumor location (sigma $85 \%$, rectum $50 \%$ ), whereas local CRC recurrence has a poor prognosis without reported long-term survival [23•]. Overall 3-year survival is reported to be $40 \%[1 \bullet \bullet$.

\section{Gynecological Tumors}

Gynecological tumors comprise endometrial cancer, cervical cancer, ovarian cancer, vulvar, and vaginal malignancies. Endometrial cancer is the most common gynecological malignancy in the developed world.

Advanced primary disease, tumor recurrence or complications following cancer treatment can involve the 
Fig.. 5 a MRI T2 sequence in axial plane, $\mathbf{b}$ fused MRI-PET image in axial plane, $\mathbf{c}$ PET, d MRI T2 sequence in sagittal plane. Female patient (68y) with cervical cancer, post-

hysterectomy and extensive recurrence with tumor invasion of the bladder, urethra and the vagina and with vesico-vaginal fistula
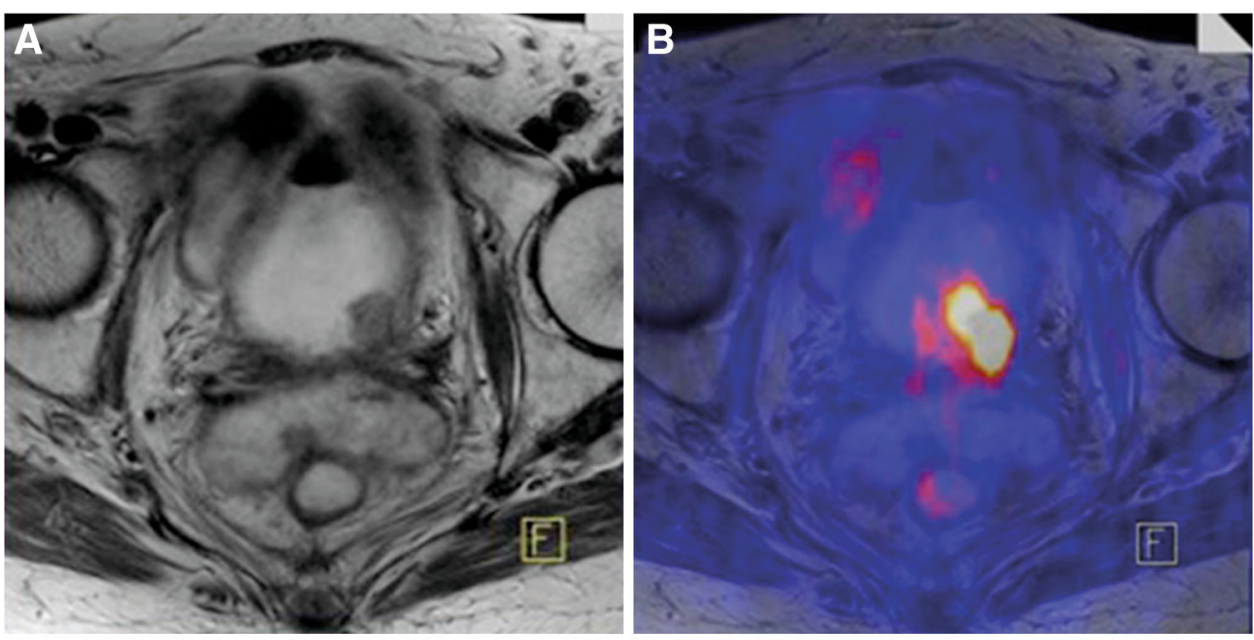

C

D

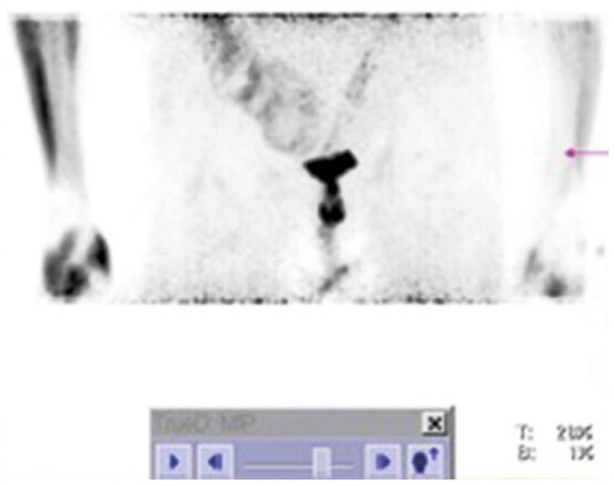

urinary tract. In an advanced stage (IIIB) cervical cancer extends to the pelvic wall causing hydronephrosis. Cervical, endometrial, and vaginal cancer can involve the bladder in stage IVA. In advanced stages, vulvar cancer spreads to the lower urethra (IIIi) and invades the upper urethra and/or the bladder mucosa (IVA).

Treatment of advanced or recurrent cervical cancer will depend on previous treatment, site, or extent of recurrence, disease-free interval, and patient's performance status at presentation. Radiation therapy in combination with chemotherapy may cure $40-50 \%$ of patients with recurrence in the pelvis following radical surgery. Pelvic exenteration is considered a treatment option for central pelvic recurrence after prior radiation therapy.

Central pelvic recurrences of endometrial cancer are treated with pelvic radiotherapy with intent to cure if there is no evidence of distant metastatic disease. Palliative treatment comprises radiotherapy, chemotherapy, and hormonal therapy and is targeted at the symptom, taking into account previous treatment and patient factors.

Patients with an advanced stage of ovarian cancer are treated with a combination of cytoreductive surgery and chemotherapy. Neoadjuvant chemotherapy and interval debulking for patients in stage III or IV, who are not good surgical candidates up front (e.g., large pleural effusion), should be considered.

If it is possible to resect the primary vulvar lesion with clear surgical margins and without sphincter damage that could cause urinary incontinence, primary surgical excision is desirable. If primary surgery would result in the need for a urinary stoma, it is preferable to employ primary radiation therapy, followed by a more limited resection of the tumor bed. Chemoradiation has been used, sometimes without the need for surgical resection of the tumor bed.

Possible urinary complications following gynecological treatment are: immediately reparable intraoperative injury, residual volume of urine, permanent urinary retention, urinary incontinence, cystitis, urethral stenosis, vesicovaginal fistulas, uretero-vaginal fistulas, with subsequent inadequate renal function or non-functioning kidney, which may require nephrectomy or permanent nephrostomy. Radiation morbidity following treatment for gynecological cancer comprises frequency of urination or nocturia, dysuria, hematuria, and reduced bladder capacity. [9••] 


\section{Sarcomas}

Soft tissue sarcomas are in general a heterogeneous group of tumors, often with no recognized "gold standard" for care. They are relatively uncommon, account for approximately $1 \%$ of adult cancers [29] and arise from different mesenchymal cells. There are more than 50 separate histological subtypes with different biological behavior and prognosis, divided into two broad categories: soft tissue sarcomas and sarcomas of the bone [30]. The pelvis as one localization of these tumors represents a complex space with multiple vital structures in close relationship. Nevertheless, the gastrointestinal and urinary tracts are often displaced, they are rarely invaded and gastrointestinal or urinary symptoms are unusual. Because of their rarity, relatively late presentation due to the inaccessibility of the region and their usually substantial size before symptoms appear, soft tissue sarcomas present several therapeutic challenges.

If radiological characteristics of the pelvic sarcoma are in doubt and neoadjuvant treatment may be appropriate, a computer-guided core needle biopsy is required as induction therapy. Moreover, a biopsy may be indicated if the tumors, particularly lymphomas or sarcomas with distant metastases, require chemotherapy.

Complete surgical resection is the only potentially curative treatment modality for pelvic as well as all retroperitoneal sarcomas. However, more than the tumors arising from the urinary, gastrointestinal, or gynecological pelvic tract, local recurrence occurs in a large proportion of patients and is responsible for as much as $75 \%$ of sarcoma-related deaths [31]. More than in other tumors, complete macroscopic resection is therefore the most important factor in avoiding local recurrence and ultimately promoting overall survival. Although at first presentation tumors are generally well encapsulated, their large diameter and anatomical contact to adjacent organs in the pelvis usually require mutilating surgery with en bloc resection of contiguous organs like ureter, bladder, rectum/ colon, uterus/adnexes to achieve a complete margin-negative surgical resection. Good and detailed information must be provided to the patient about the surgical procedure, morbidity, and the influence of the procedure on quality of life and survival. At the time of local recurrence, the possibility to obtain negative margins is significantly smaller and each successive operation becomes more difficult than the previous one. In these cases surgery is only a matter of symptom control.

In an attempt to lessen local recurrence there is currently an increase in multidisciplinary specialization in neoadjuvant chemotherapy and/or radiotherapy according to histological subtype in high-volume centers in order to ensure short-term surgical outcome and superior long-term local recurrence and overall survival rates.
Figure 6 shows the CT imaging of a 60 -year-old male with a sarcoma that arose from the pelvis with pulmonary metastases.

\section{Resection and Reconstruction}

\section{Ureteral Reconstruction}

In cases of ureter resection or iatrogenic injury different techniques are available to reestablish urine transport depending on the location and the length of the defect. Ureteroureterostomy is feasible for short defects of the upper- and mid-ureter. Clean and well vascularized resection margins as well as spatulation of the ends with double$\mathrm{J}$ stenting are essential for a success rate of up to $90 \%$ [5, 32]. Distal ureteral injury close to the urinary bladder can be managed by direct reimplantation. If a tension-free ureterocystoneostomy is not achievable, a combination of either a psoas hitch (Fig. 7) or a Boari flap is possible. Psoas hitch and Boari flap (urinary bladder flap rotated upwards) are also the methods of choice for covering longer distances up to $15 \mathrm{~cm}$ with success rates of at least $85 \%$ [5, 33]. Ileal ureteral substitution enables reconstruction of the total distance; kidney autotransplantation should be reserved for selected cases [5].

\section{Bladder-Sparing Resection}

Infiltrating nonurogenital cancers are characterized by unifocal involvement and thus bladder-sparing surgery is oncologically justified if negative margins can be obtained [1••]. Feasibility of planned bladder-sparing resection should ultimately be based on intraoperative findings and in most patients will result in preservation of quality of life $[1 \bullet, 34]$. In addition to oncological resection, ureteral reimplantation will be necessary if the bladder trigone is involved. Furthermore, enterocystoplasty using terminal ileum is an option if bladder capacity after extensive resection is diminished and cystectomy is not necessary from an oncological point of view [35••]. Patients generally have to be informed about possible consequences after bladder-sparing surgery (e.g., residual urine, overactive bladder), which is generally treated according to the guidelines for a neurogenic bladder [36].

\section{Cystectomy, Multivisceral Resection, Pelvic}

\section{Exenteration}

Extensive radical surgery is a surgical option for extensive pelvic masses/infiltration if either negative margins can be achieved or the extent of the surgery is justified by palliation of the patient, especially in situations involving huge 
Fig. 6 Cross- $(\mathbf{a}, \mathbf{b})$ and coronal section (c): CT image of a 60-year-old male showing a huge central pelvic mass with pulmonary metastases

(d) arising from the pelvis. The mass measured

$15 \times 10 \times 10 \mathrm{~cm}$ in diameter and weighed $712 \mathrm{~g}$. It was displacing and extremely compressing the rectum to the left side and the bladder anteriorly, but not invading. Indication for operation was bowel obstruction. Histology was consistent with a pleomorph, low-grade sarcoma of the pelvis
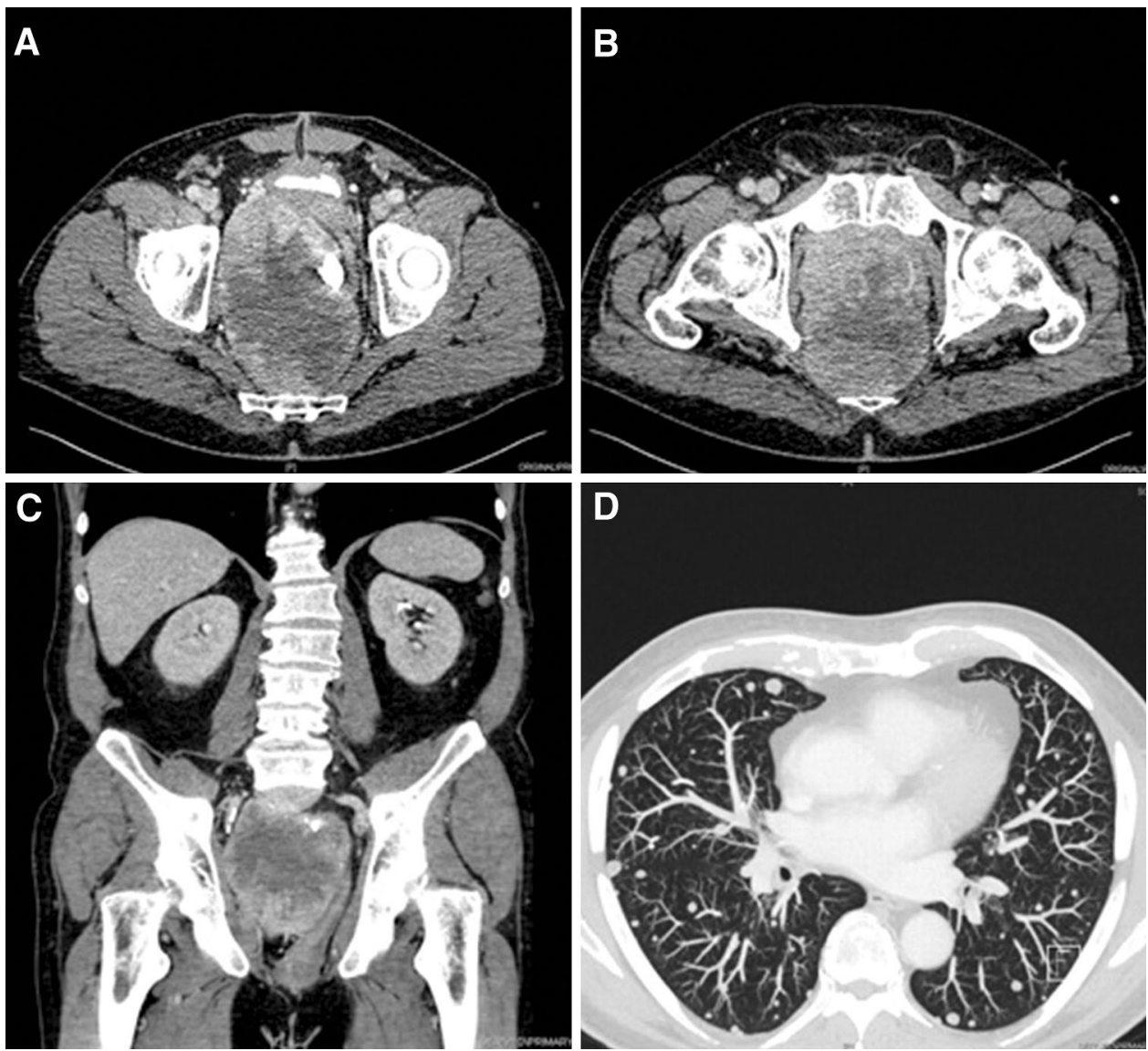
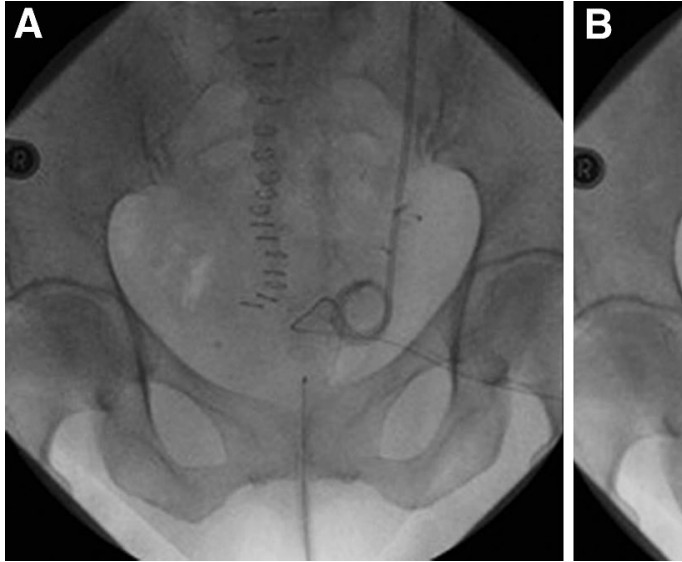

Fig. 7 a Conventional X-ray of a patient after ureteric reimplantation with psoas hitch as part of the treatment for retroperitoneal recurrence of colorectal cancer. The image demonstrates a transurethral, a suprapubic and a left double-J catheter. b Cystography illustrates the

tumor necrosis leading to fistulas. The extent of radical surgery can be anticipated from preoperative imaging in some patients, but more often intraoperative findings guide the surgeon to the necessary strategy. Despite the adverse effects for the patients, 5-year survival rates of up to $66 \%$ have been reported, depending on the underlying primary cancer [37].

In male patients, resection of the urinary bladder is usually performed with simultaneous prostatectomy, especially if prostate infiltration is suspected [4, 5, 38]. 
Fig. 8 a Cystography after orthotopic neobladder reconstruction 14 days after surgery (volume about $80 \mathrm{ml}$ ). The Kerckring folds are still visable. b Same patient as image a 3 months after surgery. The volume has increased to a physiological „bladder“volume of 300-400 ml. c Patient with prostate-sparing cystectomy and orthotopic neobladder with micturition problems (residual urine) due to a crossing fold on the left side. d CT image of the same patient illustrates the fold acting as a valve mechanism during micturition effort
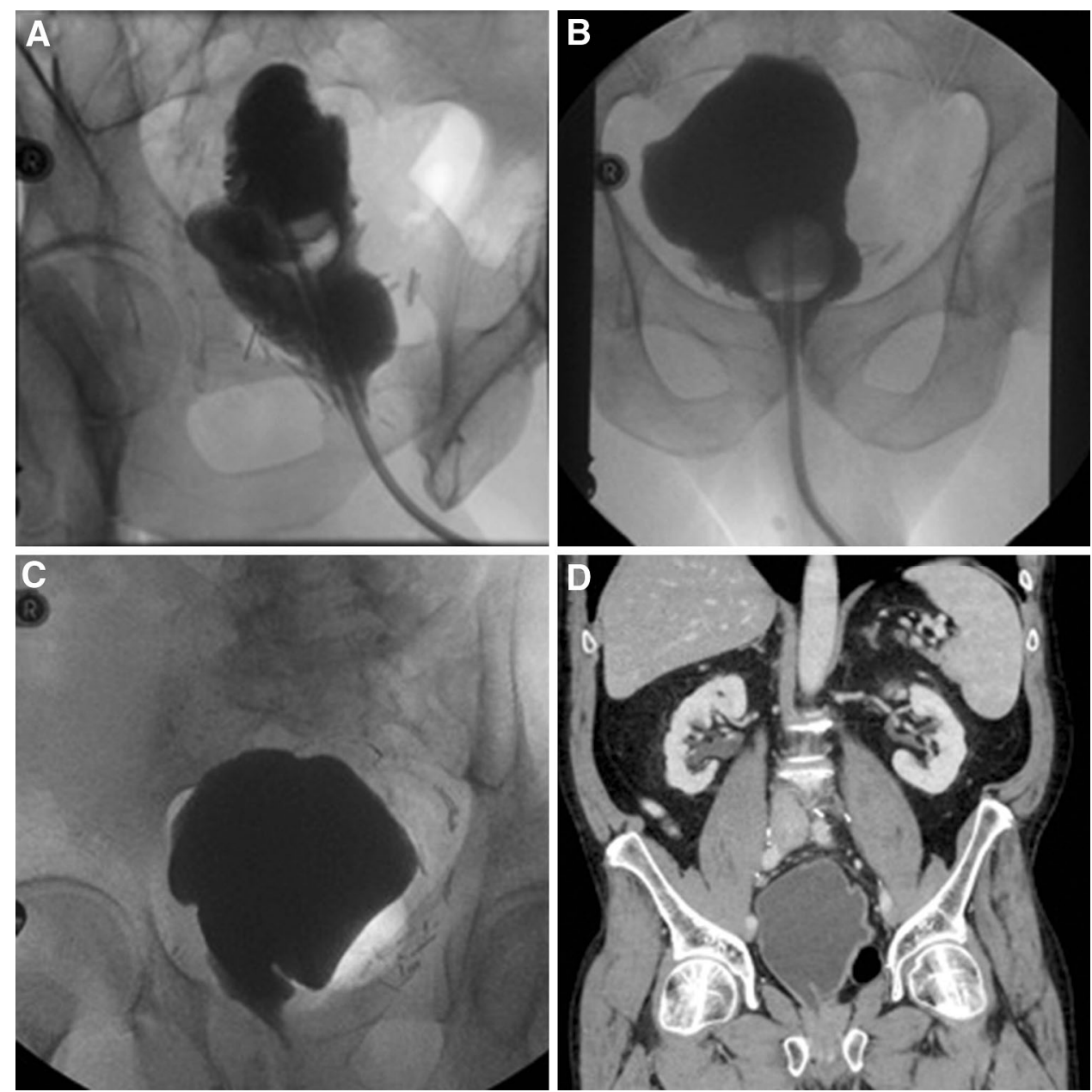

Data for urethrectomy in nonurological pelvic cancer do not exist. Extirpation of the urethra should be considered in both genders if pelvic floor infiltration is histologically proven or highly suspected, although orthotopic neobladder reconstruction will be impossible in these cases.

Nerve sparing of autonomic nerve courses surrounding the prostate or the vagina, which are responsible for erectile function and partly urinary continence, is currently of great interest in radical bladder cancer treatment [39]. In contrast to bladder cancer, extensive pelvic surgery (e.g., cystectomy, exenteration) for nonurological cancer is necessary in patients with large tumor burden, for which reason preservation of neurovascular structures is frequently impossible. In addition, neoadjuvant or adjuvant radiotherapy or chemotherapy further impacts neural function, even if preservation has been carried out.

The dimension of resection of the female inner genitalia and the extent of rectal surgery ((low) anterior resection vs. rectum amputation) ultimately also depends on intraoperative findings with the primary goal of achieving negative surgical margins.
Histopathology

With regard to the required en bloc resection of the pelvic mass, intraoperative frozen sections should be retrieved after removal of the whole specimen, to avoid intra-abdominal tumor cell seeding. Frozen sections might be helpful in determining negative margins intraoperatively, which allows the possibility of further resection, if necessary $[1 \bullet \bullet]$.

Prasad et al. describe a surgical safety margin of $2 \mathrm{~cm}$ in their series of nonurological pelvic tumors, which was confirmed by frozen section together with final histopathology $[1 \bullet \bullet$.

\section{Urinary Diversion}

Over the years, a variety of continent and incontinent urinary diversions have been developed $[4,5]$. To date, orthotopic neobladder, ileal conduit, ureterocutaneostomy and, in selected patients, continent cutaneous reservoirs have been established as effective options to urine collection or diversion $[4,40]$. 
Intact function of the external urinary sphincter complex is essential for the placement of an orthotopic neobladder, which is typically formed from a terminal ileal segment. Several authors also prefer other intestinal segments (e.g., orthotopic ileocecal ascending colon reservoirs), which have demonstrated their efficacy [41]. Candidates for orthotopic neobladders are characterized by negative surgical margins, young patient age, acceptance of impossibility of pelvic radiotherapy, acceptance of possible urinary incontinence and, in cases with residual urine, the practice of aseptic intermittent catheterization (Fig. 8) [5]. Since the last decade, neobladder reconstruction has been established in female patients and shows results comparable to those in male studies with a mild increase in urinary retention [42]. Main advantage of the choice of a neobladder is preservation of body image, but patients need time to increase the volume of the neobladder, to control daily continence and to accept the increased risk of nocturnal incontinence (Fig. 8) [4].

Incontinent cutaneous urinary diversions, namely the ileal conduit, are characterized as procedures performed quickly and relatively easily. Besides compromised body image, patients have to learn urostomy handling. Advantages are faster recovery, the possibility to also treat aged patients, and the potential for postoperative pelvic radiotherapy. Side-effects from using bowel segments for urinary diversion are uncommon in contrast to neobladder patients due to the shorter length of diverted intestine (10-15 cm for ileal conduit vs. 40-100 cm, depending on the kind of neobladder) [4, 38].

Although ureterocutaneostomy (unilateral, bilateral, or transuretero-ureterostomy) is a very traditional procedure, it remains a reasonable option in patients with reduced medical condition. The main advantage is the urinary diversion without the use of bowel segments and therefore, the reduced risk for otherwise necessary intestinal anastomosis [43]. Patients must be informed about the increased risk of stenosis, which might call for permanent stenting with regular changes [4].

In selected patients with impaired function of the urinary sphincter but good medical condition and oncological prognosis, the continent cutaneous urinary diversion with detubularized intestinal segments (ileal, colonic or combinations) can be discussed. Patients should be informed about the regular (4-6 times a day) catheterization of the pouch and the increased risk for metabolic acidosis, urinary tract infection, and mucus/stone formation in contrast to incontinent conduits [44•].

In summary, the possible urinary diversion options should be discussed with the patient preoperatively and the patient's preference noted, but informed consent must be obtained for both continent and incontinent diversion, depending on the extent of resection.

\section{Conclusion}

Nonurological pelvic tumors infiltrating the lower urinary tract are almost always colorectal cancer, gynecological tumors, or sarcomas. Tumor infiltration often cannot be distinguished from inflammatory adhesions preoperatively or intraoperatively. Comprehensive diagnostics, extensive informed consent of the patient about planned procedures and possible expansions of the resection and subsequent reconstruction, as well as interdisciplinary discussion and preparation of the intervention, are essential to ensure optimal oncological outcome and preferably high quality of life. The extent of surgical resection depends on several factors. The goal of complete resection with negative surgical margin and sufficient safety length seem to be the most import criteria. For this reason, en bloc resection should be aimed for, which in some cases requires even a multivisceral resection or total exenteration with potential loss of organ function. Patient age, general condition, oncological prognosis and, finally, the patient's personal decision regarding acceptance of the surgical scope with possibly persistent disability should be given consideration.

\section{Compliance with Ethics Guidelines}

Conflict of Interest Claudius Falch, Bastian Amend, Sven Müller, Andreas Kirschniak, Ruth Ladurner, Beate Wietek, Karl-Dietrich Sievert, Christl Reisenauer, and Alfred Königsrainer declare no conflicts of interest.

Human and Animal Rights and Informed Consent This article does not contain any studies with human or animal subjects performed by any of the authors.

\section{References}

Papers of particular interest, published recently, have been highlighted as:

- Of importance

- Of major importance

1. • Siva Prasad G, et al. Bladder-sparing surgery in locally advanced nonurological pelvic malignancy. Urol Int. 2006;77(1):18-21. Validation of bladder-sparing surgery in 15 patients with locally advanced nonurological pelvic malignancy. Presentation of tumor entities, localization of infiltration, diagnosis, intraoperative findings and outcome.

2. Chen YG, et al. Adhesion pattern and prognosis studies of T4N0M0 colorectal cancer following en bloc multivisceral resection: evaluation of T4 subclassification. Cell Biochem Biophys. 2011;59(1):1-6.

3. Stubinger SH, van der Horst C, Braun PM. Pelvic tumors in the eyes of urologists. Ther Umsch. 2007;64(7):395-8.

4. Witjes JA, et al. EAU guidelines on muscle-invasive and metastatic bladder cancer: summary of the 2013 guidelines. Eur Urol. 2014;65(4):778-92. 
5. Wein AJ, et al. Campbell-Walsh Urology. 9th ed. Philadelphia: W.B. Saunders; 2007.

6. Chuttiangtum A, Udomthavornsuk B, Chumworathayi B. Hematuria screening test for urinary bladder mucosal infiltration in cervical cancer. Asian Pac J Cancer Prev. 2012;13(10):4931-3.

7. Lokeshwar VB, et al. Bladder tumor markers beyond cytology: International Consensus Panel on bladder tumor markers. Urology. 2005;66(6 Suppl 1):35-63.

8. Snyman LC, et al. Urine cytology as a screening test for bladder infiltration in cervical cancer. Int J Gynecol Cancer. 2006;16(4): 1587-90.

9. •- Best Clinical Practice. Gynaecological Cancer Guidlines. 2009. Current guideline on gynaecological cancer.

10. Sibai $\mathrm{H}$, et al. Hydronephrosis with diffuse or segmental cortical thinning: impact on renal function. J Urol. 2001;165(6 Pt 2):2293-5.

11. Yaman O, et al. Staging with computed tomography, transrectal ultrasonography and transurethral resection of bladder tumour: comparison with final pathological stage in invasive bladder carcinoma. Br J Urol. 1996;78(2):197-200.

12. Huang WC, et al. Ultrasonographic characteristics and cystoscopic correlates of bladder wall invasion by endophytic cervical cancer. Ultrasound Obstet Gynecol. 2006;27(6):680-6.

13. Taghizadeh AK, et al. When does it hurt? Pain during flexible cystoscopy in men. Urol Int. 2006;76(4):301-3.

14. Leicht W, et al. Colovesical fistula caused by diverticulitis of the sigmoid colon: diagnosis and treatment. Urologe A. 2012;51(7): 971-4.

15. Forstner R. Radiological staging of ovarian cancer: imaging findings and contribution of CT and MRI. Eur Radiol. 2007; 17(12):3223-35.

16. Viswanathan AN, Buttin BM, Kennedy AM. Oncodiagnosis Panel: 2006. Ovarian, cervical, and endometrial cancer. Radiographics. 2008;28(1):289-307.

17. Kyriazi S, et al. Diffusion-weighted imaging of peritoneal disease for noninvasive staging of advanced ovarian cancer. Radiographics. 2010;30(5):1269-85.

18. Whittaker CS, et al. Diffusion-weighted MR imaging of female pelvic tumors: a pictorial review. Radiographics. 2009;29(3): 759-74 discussion 774-778.

19. Gu P, et al. CA 125, PET alone, PET-CT, CT and MRI in diagnosing recurrent ovarian carcinoma: a systematic review and meta-analysis. Eur J Radiol. 2009;71(1):164-74.

20. Yen TC, et al. Defining the priority of using 18F-FDG PET for recurrent cervical cancer. J Nucl Med. 2004;45(10):1632-9.

21. Mohan HM, et al. Multivisceral resection in colorectal cancer: a systematic review. Ann Surg Oncol. 2013;20(9):2929-36.

22. Kobayashi $\mathrm{T}$, et al. High incidence of urinary bladder involvement in carcinomas of the sigmoid and rectum: a retrospective review of 580 patients with colorectal carcinoma. J Surg Oncol. 2003;84(4):209-14.

23. - Fujisawa M, et al. Surgical management of the urinary tract in patients with locally advanced colorectal cancer. Urology. 2002;60(6):983-7. Evaluation of patients with carcinoma in the sigmoid colon, rectum or local recurrence after performing bladder-sparing procedure or cystectomy.

24. Balbay MD, et al. Rationale for bladder-sparing surgery in patients with locally advanced colorectal carcinoma. Cancer. 1999;86(11):2212-6.

25. - Pox C, et al. S3-guideline colorectal cancer version 1.0. Z Gastroenterol. 2013;51(8):753-854. Current guideline on colorectal carcinoma.
26. Bujko K, et al. Sphincter preservation following preoperative radiotherapy for rectal cancer: report of a randomised trial comparing short-term radiotherapy versus conventionally fractionated radiochemotherapy. Radiother Oncol. 2004;72(1):15-24.

27. Cahill RA, Leroy J, Marescaux J. Could lymphatic mapping and sentinel node biopsy provide oncological providence for local resectional techniques for colon cancer? A review of the literature. BMC Surg. 2008;8:17.

28. Yang TX, Morris DL, Chua TC. Pelvic exenteration for rectal cancer: a systematic review. Dis Colon Rectum. 2013;56(4):519-31.

29. Jemal A, et al. Cancer statistics, 2007. CA Cancer J Clin. 2007;57(1):43-66.

30. Grimer R, et al. Guidelines for the management of soft tissue sarcomas. Sarcoma. 2010;2010:506182.

31. Strauss DC, Hayes AJ, Thomas JM. Retroperitoneal tumours: review of management. Ann R Coll Surg Engl. 2011;93(4): 275-80.

32. Hackethal A, et al. Feasibility and outcomes of ureteroureterostomy and extravesical ureteroneocystostomy as part of radical surgery for infiltrating gynecologic disease. Int J Gynecol Cancer. 2013;23(6):1139-45.

33. Rafique M, Arif MH. Management of iatrogenic ureteric injuries associated with gynecological surgery. Int Urol Nephrol. 2002; 34(1):31-5.

34. Gao F, et al. Outcome of surgical management of the bladder in advanced colorectal cancer. Int J Colorectal Dis. 2007;22(1):21-4.

35. $\bullet$ Winter DC, et al. Local involvement of the urinary bladder in primary colorectal cancer: outcome with en-bloc resection. Ann Surg Oncol. 2007;14(1):69-73. The publication shows the importance of a radical en-bloc resection including parts or the complete urinary bladder in locally advanced colorectal cancer for achieving a better oncological outcome.

36. Vidart A, et al. Neurourological consequences of gynaecological surgery (endometriosis, simple hysterectomy, radical colpohysterectomy), colorectal surgery and pelvic radiotherapy. Prog Urol. 2007;17(3):381-4.

37. Heger U, et al. [Total pelvic exenteration]. Chirurg. 2010;81(10): 897-901.

38. Smith JA, et al. Hinman's atlas of urologic surgery. Philadelphia: W.B. Saunders; 2012.

39. Modh RA, Mulhall JP, Gilbert SM. Sexual dysfunction after cystectomy and urinary diversion. Nat Rev Urol. 2014;11:445-53.

40. Lee RK, et al. Urinary diversion after radical cystectomy for bladder cancer: options, patient selection, and outcomes. BJU Int. 2014;113(1):11-23.

41. Ungar L, Palfalvi L. Pelvic exenteration without external urinary or fecal diversion in gynecological cancer patients. Int J Gynecol Cancer. 2006;16(1):364-8.

42. Stenzl A, et al. Urethra-sparing cystectomy and orthotopic urinary diversion in women with malignant pelvic tumors. Cancer. 2001;92(7):1864-71.

43. Pycha A, et al. Comparison of complications in three incontinent urinary diversions. Eur Urol. 2008;54(4):825-32.

44. - Urh A, et al. Postoperative outcomes after continent versus incontinent urinary diversion at the time of pelvic exenteration for gynecologic malignancies. Gynecol Oncol. 2013;129(3):580-5. Data of urinary diversion after exenteration, performed for pelvic malignancies are rare. The authors report on a cohort of 133 patients with comparable results for continent and incontinent conduits in the long term. 